were 44.3 years old and 7.0 years. Shoulder (77.9\%) was the most commonly reported affected area, followed by the lower back (75.7\%), neck (69.2\%), wrist and hand (62.5\%), ankle (59.1\%), elbow (57.2\%), knee (56.3\%) and thigh (46.2\%). In work-related risk factors, $60 \%$ NAs reported more than 8 hours standing per day, and $34.3 \%$ NAs had to lifted over 10 $\mathrm{Kg}$ heavy more than 20 times a day. $37.7 \%$ NAs reported that they were injuried or had disease from this job in this year. The presence of shoulder pain was significantly related to tenure (OR 2.3), awkward postures (OR 2.5), inadequate height of work desk/chair (OR 3.2). Low back pain was related to awkward postures (OR 2.7) and standing hours per day (OR 4.7). Neck pain was related to awkward postures (OR 2.3) and inadequate height of work desk/chair (OR 3.1). Wrist and hand pain was related to awkward postures (OR 3.7).

Conclusion The prevalence of LBP among NAs in Taiwan is high. Any MSD reduction interventions that aim to improve ergonomic and work environments for NAs should take this information into consideration.

\section{RF-424 SICKNESS ABSENCE AND MECHANICAL AND PSYCHOSOCIAL WORK EXPOSURES ACROSS OCCUPATIONAL GROUPS IN NORWAY}

'Karina Undem, Ingrid Sivesind Mehlum, Svetlana Solovieva, Therese N Hanvold, Petter Kristensen, Taina Leinonen. ' National Institute of Occupational Health, Norway

\subsection{6/OEM-2021-EPI.360}

Introduction The working environment may contribute strongly to the development and manifestation of health problems leading to reduced work participation. To maintain high workforce participation, it is important to target workplace interventions to occupational groups at high risk of sickness absence (SA).

Objectives To identify occupational groups with excessive SA and develop occupation-specific knowledge about the contribution of work-related factors to SA and the potential for prevention.

Methods We performed a register-based study on employees aged $25-59$ in $2013(\mathrm{~N}=1,331,547)$ and calculated genderand occupation-specific (4-digit ISCO codes) one-year incidence of all-cause and cause-specific SA. We selected the following job exposures: heavy physical work, high job demands and low job control, assessed by a Job Exposure Matrix and compared the gender-specific risk of SA among exposed workers to non-exposed workers. Lastly, we compared the genderspecific risk of SA in ten occupational groups to professionals (reference), controlling for (i) age and (ii) age and job exposures. We used Cox proportional hazards model for all analyses.

Results Workers exposed to heavy physical work or low job control had higher risk of SA $(\mathrm{RR}=3.65 ; 95 \%$ CI $3.54-3.78$ and $\mathrm{RR}=1.41 ; 95 \%$ CI $1.39-1.42$, respectively). The ten selected occupational groups all had higher risk of SA, relative to professionals. The relative risk was particularly high among male drivers and mobile plant operators $(\mathrm{RR}=2.57$; 95\% CI 2.49-2.64) and female personal care workers $(\mathrm{RR}=1.43 ; 95 \%$ CI 1.41-1.45). Adjusting for job exposures resulted in attenuation of the RR estimates, most for male building and related trade workers $(37 \%$ attenuation) and female personal care workers $(84 \%)$.
Conclusion We identified occupational groups with high risk of SA and the selected job exposures. Excess risk of SA in ten selected occupational groups, as compared to professionals, could partly be attributed to the job exposures.

\section{RF-4 INTER-RATER RELIABILITY OF OCCUPATIONAL EXPOSURE ASSESSMENT IN A CASE-CONTROL STUDY}

${ }^{1}$ E Batisse, F Labrèche, MS Goldberg, R Pasquet, J Lavoué, J Siemiatycki, ME Parent, V Ho. ${ }^{1}$ Centre de recherche du Centre hospitalier de I'Université de Montréal, Canada

\subsection{6/OEM-2021-EPI.36}

Objective To estimate inter-rater reliability of expert assessment of occupational exposures.

Methods A population-based case-control study conducted in Montreal was used to obtain detailed information on lifetime occupational histories. Two trained industrial hygienists assessed the 4,362 reported jobs to assign exposures using a checklist of 258 agents. The jobs were divided between the two experts for evaluation (initial coding); then, each reviewed the others' to reach a consensus. A job was considered 'exposed' to an agent if that agent was present at levels above the non-occupational environment. Experts rated exposure for each job/substance combination according to confidence that the exposure occurred (possible, probable, definite), and to concentration (low, medium, high), where, low and high represented the extremes in the range of levels encountered in a work environment. An inter-rater reliability sub-study was conducted among a random sample of 185 jobs. Each expert coded the 185 jobs (1st coding); then, 6 months later, a 2nd coding occurred, whereby each expert coded the other's evaluation but did not have access to their own 1st evaluation. The statistical unit of observation was each job/substance decision $(185$ jobs $\times 258$ substances $=47,730$ decisions/expert $)$. Chance-corrected weighted kappa statistic and Gwet's AC1 estimated the concordance between the experts in the 1st and 2nd coding.

Results Over 98\% agreement was found and $>97 \%$ $(n=36,497)$ of decisions were to attribute no exposure to a particular job/substance combination by both experts. Restricting to combinations rated as exposed by both experts $(\mathrm{n}=508), \quad$ Kappa $=0.44$ (95\%CI: $0.37-0.50)$ and Gwet $=0.55$ $(0.48-0.61)$ was found for confidence; while, Kappa $=0.30$ $(0.15-0.45)$ and Gwet=0.92 $(0.90-0.95)$ was found for concentration. After the 2nd coding, agreement improved for both confidence $(\mathrm{Kappa}=0.68,0.63-0.73$; Gwet $=0.70$, 0.65$0.75)$ and concentration $(\mathrm{Kappa}=0.65,0.50-0.80$; Gwet $=0.96$, 0.95-0.98).

Conclusion This sub-study provides some evidence supporting the reliability of expert assessment of occupational exposures in large-scale epidemiologic studies.

\section{RF-285 THE EXPOSURE RESPONSE RELATION BETWEEN OCCUPATIONAL EXPOSURE TO WOOD DUST AND SINONASAL CANCER}

${ }^{1}$ Marie Kempf Frydendahl, Theresa Maria Møller Kynde, Henrik Albert Kolstad, Inge Brosbø Iversen, Vivi Schlünssen, Signe Hjuler Boudigaard, Jens Peder Ellekilde Bonde, Jesper Medom Vestergaard, Esben Meulengracht Flachs, Ioannis Basinas. 'Aarhus University Hospital, Denmark

10.1136/OEM-2021-EPI.362 
Introduction Occupational exposure to wood dust is associated with increased risk of adenocarcinoma and probably also squamous cell sinonasal cancer. There is, however, still limited knowledge about the exposure-response relation and possible threshold levels.

Objectives To analyze the exposure-response relation between quantitative measures of wood dust exposure and risk of sinonasal cancer.

Methods A cohort study was conducted of the entire Danish working population 1979-2015 $(\mathrm{n}=5,421,248)$ with annual information on occupation since 1977. An inception population of workers 20 years or younger in 1977 was also established $(n=3,012,247)$. Annual wood dust levels were assigned to each participant with a quantitative job exposure matrix (JEM) modelled from 12,653 personal measurements. A total of 2,576 incident cases of sinonasal cancer were identified in the National Patient Register during follow up. Incidence rate ratios (IRR) were analyzed in discrete time hazard models adjusted for gender, age, calendar year, education and JEM estimates of smoking probability.

Results During 36 years of follow up we identified 309 wood dust exposed cases. The adjusted IRR (95\%CI) was 1.83 (1.56-2.15) for the highest cumulative exposure tertile ( $>11.2$ $\mathrm{mg} / \mathrm{m}^{3}$-years), $1.66(1.41-1.97)$ for the longest duration of exposure (>5 years), and $1.64(1.38-1.95)$ for the highest mean exposure tertile $\left(>2.06 \mathrm{mg} / \mathrm{m}^{3}\right)$ compared with no exposure. Trend tests were statistically significant only for mean exposure $(\mathrm{P}>0.001)$. No increased risk of sinonasal cancer was observed in the inception cohort that, however, only included 28 wood dust exposed cases.

Conclusion We found increased risk of sinonasal cancer associated with high-level wood dust exposure, but with no consistent trends. Future analyses of this material should separate adenocarcinomas from other histological subtypes.

\section{RF-287 THE OCCUPATIONAL ENVIRONMENT AND OVARIAN CANCER RISK}

${ }^{1}$ Lisa Leung, Pascal Guenel, Anita Koushik. 'Université Paris-Saclay (CESP, Inserm), France and Université de Montréal (CRCHUM), Canada

\subsection{6/OEM-2021-EPI.363}

Introduction High-quality epidemiological evidence on the relationship between the occupational environment and ovarian cancer risk is limited. Most studies had very few cases, did not adjust for important confounders, and lacked information on specific workplace exposures.

Objectives To investigate the relationship between occupation, specific workplace exposures, and ovarian cancer risk.

Methods In a population-based case-control study conducted in Montreal, Canada (2011-2016), lifetime occupational histories were collected for 492 cases and 897 controls. For each job held by a participant, occupational and industrial classification codes were coded by an industrial hygienist. To identify specific workplace exposures, job codes were linked to the Canadian job-exposure matrix. Twenty-three agents with relatively high prevalence were selected. Occupations were examined by comparing participants ever employed in a given occupation for at least ten years vs. never employed in that occupation. For specific exposures, ever exposure, exposure duration, and cumulative exposure to selected agents were analyzed. Odds ratios (OR) and 95\% confidence intervals (CI) for associations with ovarian cancer risk were estimated using logistic regression.

Results Elevated ORs were observed for accountants $(\mathrm{OR}=2.03$, 95\% CI: 1.09-3.76); hairdressers, barbers, and beauticians $(\mathrm{OR}=3.22,95 \% \mathrm{CI}$ : 1.25-8.28); sewers and embroiderers ( $\mathrm{OR}=1.89$, 95\% CI: 0.78-4.59); saleswomen, shop assistants, and demonstrators $(\mathrm{OR}=1.42$, 95\% CI: 0.70 2.89); and occupations in retail trade $(\mathrm{OR}=1.59,95 \% \mathrm{CI}$ : 1.05-2.39). For specific exposures, increased risks were suggested for cosmetic talc, ammonia, hydrogen peroxide, hair dust, propellant gases, fluorocarbons, ethanol, cellulose, and polyester fibres. Hairdressers, barbers, and beauticians were the most frequent occupation exposed to six out of nine specific workplace exposures among participants.

Conclusion Study results suggest that certain occupations may be associated with increased ovarian cancer risks, but it is difficult to determine specific exposures that may contribute to the increased risks. Future larger studies with expert assessments of specific occupational exposures may better characterize co-exposures.

\section{RF-291 LONG WORKING HOURS AND PROSTATE CANCER RISK: RESULTS FROM A POPULATION-BASED CASE-CONTROL STUDY}

${ }^{1}$ Charlotte Salmon, Marie-Elise Parent. I'Institut national de la recherche scientifique, Université du Québec, Canada

\subsection{6/OEM-2021-EPI.364}

Introduction Recent evidence suggests that long work hours have a detrimental effect on health, possibly through unhealthy lifestyle behaviours, stress, diet and sleep disturbances. Hardly any research has evaluated whether long work hours relate to cancer incidence.

Objectives To investigate the association between long work hours and prostate cancer risk, overall and by cancer aggressiveness.

Methods We used data from the Prostate Cancer \& Environment Study, a case-control study conducted in Montreal, Canada, in 2005-2012. In all, 1,931 incident cases, aged < 7 6, were ascertained across hospitals in 2005-2009 and 1,994 age-matched ( \pm 5 years) controls were randomly selected from the electoral list. Detailed descriptions of each job held for $\geq 2$ years, including working hours, were elicited through in-person interviews. Long work hours were defined as working time exceeding the Canadian standard of 40 hours/week. Unconditional logistic regression was used to derive odds ratio (ORs) and 95\% confidence intervals (CIs) for the association between long work hours and prostate cancer risk, adjusted for age, education and ancestry. We also investigated whether associations varied according to a history of work at night.

Results Overall, 2,477 subjects (64.6\%) reported ever working $>40$ hours/week. Occupations entailing long hours were most often related to management, administration and sales. Ever exposure to long work hours was associated with an OR of prostate cancer of 1.21 (95\%CI 1.06-1.39), while it was of 1.50 (95\%CI 1.23-1.83) for a cumulative duration of 11-23 years of long work hours. The ORs for aggressive cancer among men who engaged in long work hours and who had a history of work at night were 1.40 (95\%CI $0.85-2.30)$, and 1.20 (95\% CI 0.91-1.57) among those without such a history. 